\title{
On a q-analogue of the Hilbert's type inequality
}

\section{Zhengping Zhang, Gaowen Xi*}

College of Mathematics and Physics, Chongqing University of Science and Technology, Chongqing, 401331, P. R. China.

\author{
Communicated by S. H. Wu
}

\begin{abstract}
In this paper, by introducing a parameter $\mathrm{q}$ and using the expression of the beta function establishing the inequality of the weight coefficient, we give a q-analogue of the Hilbert's type inequality. As applications, a generalization of Hardy-Hilbert's inequality are obtained.
\end{abstract}

Keywords: q-Analogue, Hilbert's type inequality, weight coefficient, Hölder inequality, generalization.

2010 MSC: 05A30, 26D15.

(C)2018 All rights reserved.

\section{Introduction}

q-Series, which are also called basic hypergeometric series, play a very important role in many fields, such as affine root systems, Lie algebras and groups, number theory, orthogonal polynomials, physics, etc.. We first recall some definitions, notations, and known results in $[1,6,7]$.

The ratio $\left(1-\mathrm{q}^{\mathrm{a}}\right) /(1-\mathrm{q})$ is called a $\mathrm{q}$-number (or basic number) and we have:

$$
\lim _{\mathrm{q} \rightarrow 1} \frac{1-\mathrm{q}^{\mathrm{a}}}{1-\mathrm{q}}=\mathrm{a} .
$$

The following is the q-integral:

$$
\int_{0}^{1} f(t) d_{q} t=(1-q) \sum_{n=0}^{\infty} f\left(q^{n}\right) q^{n} \quad \text { and } \quad \int_{0}^{a} f(t) d_{q} t=a(1-q) \sum_{n=0}^{\infty} f\left(q^{n}\right) q^{n}
$$

When $f$ is continuous on $[0, a]$, it can be shown that

$$
\lim _{q \rightarrow 1} \int_{0}^{a} f(t) d_{q} t=\int_{0}^{a} f(t) d t
$$

\footnotetext{
${ }^{*}$ Corresponding author

Email address: xigaowen@163.com (Gaowen Xi)

doi: $10.22436 /$ jnsa.011.11.04
}

Received: 2017-08-26 Revised: 2018-07-23 Accepted: 2018-07-26 
The q-gamma function is defined as

$$
\Gamma_{q}(x)=\frac{(q ; q)_{\infty}}{\left(q^{n} ; q\right)_{\infty}}(1-q)^{1-x}
$$

where $0<\mathrm{q}<1$, $(\mathrm{a}: \mathrm{q})_{\infty}=\prod_{\mathrm{k}=\mathrm{o}}^{\infty}\left(1-\mathrm{aq^{k }}\right)$ and $\lim _{\mathrm{q} \rightarrow 1^{-}} \Gamma_{\mathrm{q}}(\mathrm{x})=\Gamma(\mathrm{x})$.

Analogous to the definition of the the beta function

$$
B(x, y)=\frac{\Gamma(x) \Gamma(y)}{\Gamma(x+y)}=\int_{0}^{1} t^{x-1}(1-t)^{y-1} d t
$$

the q-beta function is defined by

$$
B_{q}(x, y)=\frac{\Gamma_{q}(x) \Gamma_{q}(y)}{\Gamma_{q}(x+y)}=\int_{0}^{1} t^{x-1} \frac{(t q ; q)_{\infty}}{\left(t q^{y} ; q\right)_{\infty}} d_{q} t
$$

which tends to $B(x, y)$ as $q \longrightarrow 1^{-}$.

If $p, r>1, \frac{1}{p}+\frac{1}{r}=1, a_{n} \geqslant 0, b_{n} \geqslant 0$, for $n \geqslant 1, n \in N$ and $0<\sum_{n=1}^{\infty} a_{n}^{p}<\infty, 0<\sum_{n=1}^{\infty} b_{n}^{r}<\infty$, then

$$
\sum_{n=1}^{\infty} \sum_{m=1}^{\infty} \frac{a_{m} b_{n}}{m+n}<\frac{\pi}{\sin \left(\frac{\pi}{p}\right)}\left\{\sum_{n=1}^{\infty} a_{n}^{p}\right\}^{\frac{1}{p}}\left\{\sum_{n=1}^{\infty} b_{n}^{r}\right\}^{\frac{1}{r}}
$$

and

$$
\sum_{n=1}^{\infty} \sum_{m=1}^{\infty} \frac{a_{m} b_{n}}{\max \{m, n\}}<\operatorname{pr}\left\{\sum_{n=1}^{\infty} a_{n}^{p}\right\}^{\frac{1}{p}}\left\{\sum_{n=1}^{\infty} b_{n}^{r}\right\}^{\frac{1}{r}}
$$

where the constant $\frac{\pi}{\sin \frac{\pi}{p}}$ and pr is best possible for each inequality, respectively. Inequality (1.2) is HardyHilbert's inequality. Inequality (1.3) is a Hilbert's type inequality [2].

In [5, 10, 12], Krnic at al. gave some generalization and reinforcement of inequality (1.2). In [3], Kuang and Debnath gave a reinforcement of inequality (1.3):

$$
\sum_{n=1}^{\infty} \sum_{m=1}^{\infty} \frac{a_{m} b_{n}}{\max \{m, n\}}<\left\{\sum_{n=1}^{\infty}[p r-G(p, n)] a_{n}^{p}\right\}^{\frac{1}{p}}\left\{\sum_{n=1}^{\infty}[p r-G(r, n)] b_{n}^{r}\right\}^{\frac{1}{r}}
$$

where $G(t, n)=\frac{t+\frac{1}{3 t}-\frac{4}{3}}{(2 n+1)^{\frac{1}{t}}}>0(t=p, r)$.

In $[8,9], X i$ gave a generalization and reinforcement of inequalities (1.3) and (1.4):

$$
\begin{aligned}
\sum_{n=1}^{\infty} \sum_{m=1}^{\infty} \frac{a_{m} b_{n}}{\max \left\{m^{\lambda}, n^{\lambda}\right\}}< & \left\{\sum_{n=1}^{\infty}\left[\kappa(\lambda)-\frac{1}{3 r n^{\frac{r+\lambda-2}{r}}}\right] n^{1-\lambda} a_{n}^{p}\right\}^{\frac{1}{p}} \\
& \times\left\{\sum _ { n = 1 } ^ { \infty } \left[\kappa(\lambda)-\frac{1}{\left.\left.3 p n^{\frac{p+\lambda-2}{p}}\right] n^{1-\lambda} b_{n}^{r}\right\}^{\frac{1}{r}},}\right.\right.
\end{aligned}
$$

where $\kappa(\lambda)=\frac{p r \lambda}{(p+\lambda-2)(r+\lambda-2)}>0,2-\min \{p, r\}<\lambda \leqslant 2$.

$$
\begin{aligned}
\sum_{n=1}^{\infty} \sum_{m=1}^{\infty} \frac{a_{m} b_{n}}{\max \left\{m^{\lambda}+A, n^{\lambda}+B\right\}}< & \left\{\sum_{n=1}^{\infty}\left[\kappa(\lambda)-\frac{1}{n^{\frac{r+\lambda-2}{r}}}\left(\frac{1}{3 r}-\frac{B}{1+B}\right)\right] n^{1-\lambda} a_{n}^{p}\right\}^{\frac{1}{p}} \\
& \times\left\{\sum_{n=1}^{\infty}\left[\kappa(\lambda)-\frac{1}{n^{\frac{p+\lambda-2}{p}}}\left(\frac{1}{3 p}-\frac{A}{1+A}\right)\right] n^{1-\lambda} b_{n}^{r}\right\}^{\frac{1}{r}} .
\end{aligned}
$$


In this paper, by introducing a parameter $\mathrm{q}$ and using the expression of the beta function establishing the inequality of the weight coefficient, a q-Hardy-Hilbert's inequality is proved, that is a q-analogue of the Hardy-Hilbert's inequality. As applications, a generalization of Hardy-Hilbert's inequality are obtained.

\section{A Lemma}

First, we need the following formula of the Riemann- $\zeta$ function (see $[4,11,13])$ :

$$
\zeta(\sigma)=\sum_{k=1}^{n} \frac{1}{k^{\sigma}}-\frac{n^{1-\sigma}}{1-\sigma}-\frac{1}{2 n^{\sigma}}-\sum_{k=1}^{l-1} \frac{B_{2 k}}{2 k}\left(\begin{array}{l}
-\sigma \\
2 k-1
\end{array}\right) \frac{1}{n^{\sigma+2 k-1}}-\frac{B_{2 l} l}{2 l}\left(\begin{array}{l}
-\sigma \\
2 l-1
\end{array}\right) \frac{\varepsilon}{n^{\sigma+2 l-1}},
$$

where $\sigma>0, \sigma \neq 1, n, l \geqslant 1, n, l \in N, 0<\varepsilon=\varepsilon(\sigma, l, n)<1$. The numbers $B_{1}=-1 / 2, B_{2}=1 / 6, B_{3}=0$, $\mathrm{B}_{4}=-1 / 30, \ldots$ are Bernoulli numbers. In particular, $\zeta(\sigma)=\sum_{\mathrm{k}=1}^{\infty} \frac{1}{\mathrm{k}^{\sigma}}(\sigma>1)$.

Since $\zeta(0)=-1 / 2$, then the formula of the Riemann- $\zeta$ function (2.1) is also true for $\sigma=0$.

Lemma 2.1. If $p, r>1, \frac{1}{p}+\frac{1}{r}=1,2-\min \{p, r\}<\lambda \leqslant 2, n \geqslant 1, q>1$, and $n \in N$, then

$$
\begin{aligned}
\omega(n, \lambda, q, p)= & \sum_{k=1}^{\infty} \frac{1}{\max \left\{\left(q^{k}-1\right)^{\lambda},\left(q^{n}-1\right)^{\lambda}\right\}}\left(\frac{n}{k}\right)^{\frac{2-\lambda}{p}} \\
& <\frac{n^{1-\lambda}}{(q-1)^{\lambda}}\left[k(\lambda)+\mu(n)-\frac{1}{3 p n^{\frac{p+\lambda-2}{p}}}\right],
\end{aligned}
$$

and

$$
\begin{aligned}
\omega(n, \lambda, q, r)= & \sum_{k=1}^{\infty} \frac{1}{\max \left\{\left(q^{k}-1\right)^{\lambda},\left(q^{n}-1\right)^{\lambda}\right\}}\left(\frac{n}{k}\right)^{\frac{2-\lambda}{r}} \\
& <\frac{n^{1-\lambda}}{(q-1)^{\lambda}}\left[k(\lambda)+\mu(n)-\frac{1}{3 r n^{\frac{r+\lambda-2}{r}}}\right],
\end{aligned}
$$

where $\mathrm{k}(\lambda)=\frac{\mathrm{pr} \lambda}{(\mathrm{p}+\lambda-2)(\mathrm{r}+\lambda-2)}, \mu(\mathrm{n})=\frac{1}{2 \mathrm{n}}-\frac{\mathrm{n}^{\lambda-1}(\mathrm{q}-1)^{\lambda}}{2\left(\mathrm{q}^{\mathrm{n}}-1\right)^{\lambda}}$.

Proof. Equalities (2.2) and (2.3) define the weight coefficient. When $2-\min \{\mathrm{p}, \mathrm{r}\}<\lambda \leqslant 2$, taking $\sigma=$ $\frac{2-\lambda}{p} \geqslant 0, l=1$, in $(2.1)$, we obtain

$$
\zeta\left(\frac{2-\lambda}{p}\right)=\sum_{k=1}^{n} \frac{1}{k^{\frac{2-\lambda}{p}}}-\frac{p n^{\frac{p+\lambda-2}{p}}}{p+\lambda-2}-\frac{1}{2 n^{\frac{2-\lambda}{p}}}+\frac{2-\lambda}{12 p n^{1+\frac{2-\lambda}{p}}} \varepsilon_{1}
$$

where $0<\varepsilon_{1}<1$.

Taking $\sigma=\frac{2}{p}+\frac{\lambda}{r}, l=1$, we obtain

$$
\zeta\left(\frac{2}{p}+\frac{\lambda}{r}\right)=\sum_{k=1}^{n-1} \frac{1}{k^{\frac{2}{p}+\frac{\lambda}{r}}}+\frac{r n^{-\frac{r+\lambda-2}{r}}}{r+\lambda-2}+\frac{1}{2 n^{\frac{2}{p}+\frac{\lambda}{r}}}+\frac{p \lambda+2 r}{12 p r n^{1+\frac{2}{p}+\frac{\lambda}{r}}} \varepsilon_{2},
$$

where $0<\varepsilon_{2}<1$.

In addition,

$$
\omega(n, \lambda, q, p)=\sum_{k=1}^{\infty} \frac{1}{\max \left\{\left(q^{k}-1\right)^{\lambda},\left(q^{n}-1\right)^{\lambda}\right\}}\left(\frac{n}{k}\right)^{\frac{2-\lambda}{p}}
$$




$$
\begin{aligned}
& =\sum_{k=1}^{n} \frac{1}{\max \left\{\left(q^{k}-1\right)^{\lambda},\left(q^{n}-1\right)^{\lambda}\right\}}\left(\frac{n}{k}\right)^{\frac{2-\lambda}{p}}+\sum_{k=n+1}^{\infty} \frac{1}{\max \left\{\left(q^{k}-1\right)^{\lambda},\left(q^{n}-1\right)^{\lambda}\right\}}\left(\frac{n}{k}\right)^{\frac{2-\lambda}{p}} \\
& =\sum_{k=1}^{n} \frac{1}{\left(q^{n}-1\right)^{\lambda}}\left(\frac{n}{k}\right)^{\frac{2-\lambda}{p}}-\frac{1}{\left(q^{n}-1\right)^{\lambda}}+\sum_{k=n}^{\infty} \frac{1}{\left(q^{k}-1\right)^{\lambda}}\left(\frac{n}{k}\right)^{\frac{2-\lambda}{p}} \\
& =\frac{n^{\frac{2-\lambda}{p}}}{\left(q^{n}-1\right)^{\lambda}} \sum_{k=1}^{n} \frac{1}{k^{\frac{2-\lambda}{p}}}-\frac{1}{\left(q^{n}-1\right)^{\lambda}}+\frac{n^{\frac{2-\lambda}{p}}}{(q-1)^{\lambda}} \sum_{k=n}^{\infty} \frac{1}{\left(\frac{q^{k}-1}{q-1}\right)^{\lambda} k^{\frac{2-\lambda}{p}}} .
\end{aligned}
$$

Since $q>1$ and $\lim _{q \rightarrow 1^{+}} \frac{q^{k}-1}{q-1}=k$. Then, we have

$$
\begin{aligned}
\omega(n, \lambda, q, p) & =\sum_{k=1}^{\infty} \frac{1}{\max \left\{\left(q^{k}-1\right)^{\lambda},\left(q^{n}-1\right)^{\lambda}\right\}}\left(\frac{n}{k}\right)^{\frac{2-\lambda}{p}} \\
& <\frac{n^{\frac{2-\lambda}{p}}}{\left(q^{n}-1\right)^{\lambda}} \sum_{k=1}^{n} \frac{1}{k^{\frac{2-\lambda}{p}}}-\frac{1}{\left(q^{n}-1\right)^{\lambda}}+\frac{n^{\frac{2-\lambda}{p}}}{(q-1)^{\lambda}} \sum_{k=n}^{\infty} \frac{1}{k^{\frac{2}{p}+\frac{\lambda}{r}}} .
\end{aligned}
$$

By (2.4) and (2.5)

$$
\begin{aligned}
& \omega(n, \lambda, q, p)<\frac{n^{\frac{2-\lambda}{p}}}{\left(q^{n}-1\right)^{\lambda}}\left[\zeta\left(\frac{2-\lambda}{p}\right)+\frac{p n^{\frac{p+\lambda-2}{p}}}{p+\lambda-2}+\frac{1}{2 n^{\frac{2-\lambda}{p}}}\right] \\
& -\frac{1}{\left(q^{n}-1\right)^{\lambda}}+\frac{n^{\frac{2-\lambda}{p}}}{(q-1)^{\lambda}}\left[\frac{r n^{-\frac{r+\lambda-2}{r}}}{r+\lambda-2}+\frac{1}{2 n^{\frac{2}{p}+\frac{\lambda}{r}}}+\frac{p \lambda+2 r}{12 p r n^{1+\frac{2}{p}+\frac{\lambda}{r}}}\right] \\
& =\frac{n^{\frac{2-\lambda}{p}}}{\left(q^{n}-1\right)^{\lambda}} \zeta\left(\frac{2-\lambda}{p}\right)+\frac{n^{\frac{2-\lambda}{p}}}{\left(q^{n}-1\right)^{\lambda}} \times \frac{p n^{\frac{p+\lambda-2}{p}}}{p+\lambda-2} \\
& -\frac{1}{2\left(q^{n}-1\right)^{\lambda}}+\frac{1}{(q-1)^{\lambda}}\left[\frac{r n^{1-\lambda}}{r+\lambda-2}+\frac{1}{2 n^{\lambda}}+\frac{p \lambda+2 r}{12 p r n^{1+\lambda}}\right] \\
& =\frac{n^{\frac{2-\lambda}{p}}}{\left(q^{n}-1\right)^{\lambda}} \zeta\left(\frac{2-\lambda}{p}\right)+\frac{n^{\frac{2-\lambda}{p}}}{(q-1)^{\lambda}\left(\frac{Q^{n}-1}{q-1}\right)^{\lambda}} \times \frac{p n^{\frac{p+\lambda-2}{p}}}{p+\lambda-2} \\
& -\frac{1}{2\left(q^{n}-1\right)^{\lambda}}+\frac{1}{(q-1)^{\lambda}}\left[\frac{r n^{1-\lambda}}{r+\lambda-2}+\frac{1}{2 n^{\lambda}}+\frac{p \lambda+2 r}{12 p r n^{1+\lambda}}\right] \\
& <\frac{n^{\frac{2-\lambda}{p}}}{\left(q^{n}-1\right)^{\lambda}} \zeta\left(\frac{2-\lambda}{p}\right)+\frac{n^{\frac{2-\lambda}{p}}}{(q-1)^{\lambda} n^{\lambda}} \times \frac{p n^{\frac{p+\lambda-2}{p}}}{p+\lambda-2} \\
& -\frac{1}{2\left(q^{n}-1\right)^{\lambda}}+\frac{1}{(q-1)^{\lambda}}\left[\frac{r n^{1-\lambda}}{r+\lambda-2}+\frac{1}{2 n^{\lambda}}+\frac{p \lambda+2 r}{12 p r n^{1+\lambda}}\right] \\
& =\frac{n^{\frac{2-\lambda}{p}}}{\left(q^{n}-1\right)^{\lambda}} \zeta\left(\frac{2-\lambda}{p}\right)-\frac{1}{2\left(q^{n}-1\right)^{\lambda}} \\
& +\frac{1}{(q-1)^{\lambda}}\left[\frac{p r \lambda n^{1-\lambda}}{(p+\lambda-2)(r+\lambda-2)}+\frac{1}{2 n^{\lambda}}+\frac{p \lambda+2 r}{12 p r n^{1+\lambda}}\right]
\end{aligned}
$$

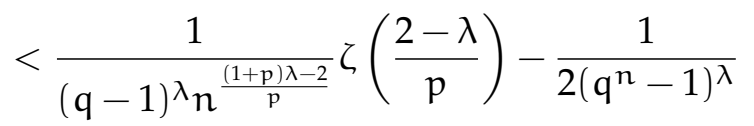

$$
\begin{aligned}
& +\frac{1}{(q-1)^{\lambda}}\left[\frac{p r \lambda n^{1-\lambda}}{(p+\lambda-2)(r+\lambda-2)}+\frac{1}{2 n^{\lambda}}+\frac{p \lambda+2 r}{12 p r n^{1+\lambda}}\right] \\
& =\frac{n^{1-\lambda}}{(q-1)^{\lambda}}\left\{\frac{p r \lambda}{(p+\lambda-2)(r+\lambda-2)}+\frac{1}{2 n}-\frac{n^{\lambda-1}(q-1)^{\lambda}}{2\left(q^{n}-1\right)^{\lambda}}\right.
\end{aligned}
$$




$$
\left.-\frac{1}{n^{\frac{p+\lambda-2}{p}}}\left[-\zeta\left(\frac{2-\lambda}{p}\right)-\frac{p \lambda+2 r}{12 p r n^{\frac{p+2-\lambda}{p}}}\right]\right\} .
$$

In (2.4), taking $n=1$, by $2-\min \{p, r\}<\lambda \leqslant 2$, we obtain

$\zeta\left(\frac{2-\lambda}{p}\right)=1-\frac{p}{p+\lambda-2}-\frac{1}{2}+\frac{(2-\lambda) \varepsilon_{1}}{12 p}<\frac{1}{2}-\frac{p}{p+\lambda-2}+\frac{2-\lambda}{12 p}=-\frac{(\lambda-2-3 p)(\lambda-2-2 p)}{12 p(p+\lambda-2)}<0$.

So for $n \geqslant 1, n \in N, 2-\min \{p, r\}<\lambda \leqslant 2$, we have

$$
\begin{aligned}
-\zeta\left(\frac{2-\lambda}{p}\right)-\frac{p \lambda+2 r}{12 p n^{\frac{p-\lambda+2}{p}}} & >\frac{(\lambda-2-3 p)(\lambda-2-2 p)}{12 p(p+\lambda-2)}-\frac{p \lambda+2 r}{12 p r} \\
& =\frac{r(\lambda-2-3 p)(\lambda-2-2 p)-(p \lambda+2 r)(p+\lambda-2)}{12 p r(p+\lambda-2)} \\
& =\frac{r(\lambda-2)^{2}+(p \lambda+5 p r+2 r)(2-\lambda)-p(p \lambda+2 r)+6 p^{2} r}{12 p r(p+\lambda-2)} \\
& >\frac{-p(p \lambda+2 r)+6 p^{2} r}{12 p r(p+\lambda-2)} \geqslant \frac{-(2 p+2 r)+6 p r}{12 r(p+\lambda-2)}>\frac{1}{3(p+\lambda-2)}>\frac{1}{3 p} .
\end{aligned}
$$

Using the last result and the inequality for $\omega(n, \lambda, q, p)$ above, we obtain (2.2).

In a similar way, one can prove (2.3).

\section{Main results}

Theorem 3.1. If $\mathrm{p}, \mathrm{r}>1, \frac{1}{\mathrm{p}}+\frac{1}{\mathrm{r}}=1, \mathrm{q}>1,2-\min \{\mathrm{p}, \mathrm{r}\}<\lambda \leqslant 2, \mathrm{a}_{\mathrm{n}} \geqslant 0, \mathrm{~b}_{\mathrm{n}} \geqslant 0$, for $\mathrm{n} \geqslant 1, \mathrm{n} \in \mathrm{N}$ and $0<\sum_{n=1}^{\infty} a_{n}^{p}<\infty, 0<\sum_{n=1}^{\infty} b_{n}^{r}<\infty$, then

$$
\begin{aligned}
\sum_{n=1}^{\infty} \sum_{m=1}^{\infty} \frac{a_{m} b_{n}}{\max \left\{\left(q^{m}-1\right)^{\lambda},\left(q^{n}-1\right)^{\lambda}\right\}}< & \frac{1}{(q-1)^{\lambda}}\left\{\sum_{n=1}^{\infty}\left[\kappa(\lambda)+\mu(n)-\frac{1}{3 r n^{\frac{r+\lambda-2}{r}}}\right]\right. \\
& \left.\times n^{1-\lambda} a_{n}^{p}\right\}^{\frac{1}{p}}\left\{\sum_{n=1}^{\infty}\left[\kappa(\lambda)+\mu(n)-\frac{1}{3 p n^{\frac{p+\lambda-2}{p}}}\right] n^{1-\lambda} b_{n}^{r}\right\}^{\frac{1}{r}},
\end{aligned}
$$

and

$$
\begin{aligned}
& \sum_{m=1}^{\infty} \frac{m^{(p-1)(\lambda-1)}}{[\kappa(\lambda)+\mu(m)]^{p-1}}\left(\sum_{n=1}^{\infty} \frac{a_{n}}{\max \left\{\left(q^{m}-1\right)^{\lambda},\left(q^{n}-1\right)^{\lambda}\right\}}\right)^{p} \\
& \quad<\frac{1}{(q-1)^{\lambda(p-1)}} \sum_{n=1}^{\infty}\left[\kappa(\lambda)+\mu(n)-\frac{1}{3 r n^{\frac{r+\lambda-2}{r}}}\right] n^{1-\lambda} a_{n}^{p}
\end{aligned}
$$

where $\mathrm{k}(\lambda)=\frac{\mathrm{pr} \lambda}{(\mathrm{p}+\lambda-2)(\mathrm{r}+\lambda-2)}>0, \mu(\mathrm{t})=\frac{1}{2 \mathrm{t}}-\frac{\mathrm{t}^{\lambda-1}(\mathrm{q}-1)^{\lambda}}{2\left(\mathrm{q}^{\mathrm{t}}-1\right)^{\lambda}}(\mathrm{t}=\mathrm{m}, \mathrm{n})$.

Proof. By Hölder inequality, we have

$$
\begin{aligned}
\sum_{n=1}^{\infty} \sum_{m=1}^{\infty} \frac{a_{m} b_{n}}{\max \left\{\left(q^{m}-1\right)^{\lambda},\left(q^{n}-1\right)^{\lambda}\right\}}= & \sum_{n=1}^{\infty} \sum_{m=1}^{\infty}\left[\frac{a_{m}}{\max \left\{\left(q^{m}-1\right)^{\lambda},\left(q^{n}-1\right)^{\lambda}\right\}^{\frac{1}{p}}}\right. \\
& \left.\times\left(\frac{m}{n}\right)^{\frac{2-\lambda}{p r}}\right]\left[\frac{b_{n}}{\max \left\{\left(q^{m}-1\right)^{\lambda},\left(q^{n}-1\right)^{\lambda}\right\}^{\frac{1}{r}}}\left(\frac{n}{m}\right)^{\frac{2-\lambda}{p r}}\right]
\end{aligned}
$$




$$
\begin{aligned}
\leqslant & \left\{\sum_{n=1}^{\infty} \sum_{m=1}^{\infty}\left[\frac{a_{m}^{p}}{\max \left\{\left(q^{m}-1\right)^{\lambda},\left(q^{n}-1\right)^{\lambda}\right\}}\left(\frac{m}{n}\right)^{\frac{2-\lambda}{r}}\right]\right\}^{\frac{1}{p}} \\
& \times\left\{\sum_{n=1}^{\infty} \sum_{m=1}^{\infty}\left[\frac{b_{n}^{r}}{\max \left\{\left(q^{m}-1\right)^{\lambda},\left(q^{n}-1\right)^{\lambda}\right\}}\left(\frac{n}{m}\right)^{\frac{2-\lambda}{p}}\right]\right\}^{\frac{1}{r}} \\
= & \left\{\sum_{m=1}^{\infty} \omega(m, \lambda, q, r) a_{m}^{p}\right\}^{\frac{1}{p}}\left\{\sum_{n=1}^{\infty} \omega(n, \lambda, q, p) b_{n}^{r}\right\}^{\frac{1}{r}} .
\end{aligned}
$$

By (2.2) and (2.3), we obtain (3.1).

By Hölder inequality and Lemma 2.1, we have

$$
\begin{aligned}
\sum_{n=1}^{\infty} \frac{a_{n}}{\max \left\{\left(q^{m}-1\right)^{\lambda},\left(q^{n}-1\right)^{\lambda}\right\}}= & \sum_{n=1}^{\infty}\left[\frac{1}{\max \left\{\left(q^{m}-1\right)^{\lambda},\left(q^{n}-1\right)^{\lambda}\right\}^{\frac{1}{p}}}\left(\frac{n}{m}\right)^{\frac{2-\lambda}{p r}}\right. \\
& \left.\times a_{n} \frac{1}{\max \left\{\left(q^{m}-1\right)^{\lambda},\left(q^{n}-1\right)^{\lambda}\right\}^{\frac{1}{r}}}\left(\frac{m}{n}\right)^{\frac{2-\lambda}{p r}}\right] \\
\leqslant & \left\{\sum_{n=1}^{\infty}\left[\frac{1}{\max \left\{\left(q^{m}-1\right)^{\lambda},\left(q^{n}-1\right)^{\lambda}\right\}}\left(\frac{n}{m}\right)^{\frac{2-\lambda}{r}} a_{n}^{p}\right]\right\}^{\frac{1}{p}} \\
& \times\left\{\sum_{n=1}^{\infty}\left[\frac{1}{\max \left\{\left(q^{m}-1\right)^{\lambda},\left(q^{n}-1\right)^{\lambda}\right\}}\left(\frac{m}{n}\right)^{\frac{2-\lambda}{p}}\right]\right\}^{\frac{1}{r}} \\
= & \left\{\sum_{n=1}^{\infty}\left[\frac{1}{\max \left\{\left(q^{m}-1\right)^{\lambda},\left(q^{n}-1\right)^{\lambda}\right\}}\left(\frac{n}{m}\right)^{\frac{2-\lambda}{r}} a_{n}^{p}\right]\right\}^{\frac{1}{p}}[w(m, \lambda, q, p)]^{\frac{1}{r}} \\
& <\left\{\sum_{n=1}^{\infty}\left[\frac{1}{\max \left\{\left(q^{m}-1\right)^{\lambda},\left(q^{n}-1\right)^{\lambda}\right\}}\left(\frac{n}{m}\right)^{\frac{2-\lambda}{r}} a_{n}^{p}\right]\right\}^{\frac{1}{p}} \\
& \times\left\{\frac{m^{1-\lambda}}{(q-1)^{\lambda}}[k(\lambda)+\mu(m)]\right\}^{\frac{1}{r}} \cdot
\end{aligned}
$$

So

$$
\begin{aligned}
& \sum_{m=1}^{\infty} \frac{m^{(p-1)(\lambda-1)}}{[k(\lambda)+\mu(m)]^{p-1}}\left(\sum_{n=1}^{\infty} \frac{a_{n}}{\max \left\{\left(q^{m}-1\right)^{\lambda},\left(q^{n}-1\right)^{\lambda}\right\}}\right)^{p} \\
& \quad<\frac{1}{(q-1)^{\lambda(p-1)}} \sum_{m=1}^{\infty} \sum_{n=1}^{\infty}\left[\frac{1}{\max \left\{\left(q^{m}-1\right)^{\lambda},\left(q^{n}-1\right)^{\lambda}\right\}}\left(\frac{n}{m}\right)^{\frac{2-\lambda}{r}} a_{n}^{p}\right]<\frac{1}{(q-1)^{\lambda(p-1)}} \sum_{n=1}^{\infty} \omega(n, \lambda, q, r) a_{n}^{p} .
\end{aligned}
$$

By Lemma 2.1, we obtain (3.2), the proof of the theorem is completed.

Corollary 3.2. If $p, r>1, \frac{1}{p}+\frac{1}{r}=1,2-\min \{p, r\}<\lambda \leqslant 2, a_{n} \geqslant 0, b_{n} \geqslant 0$, for $n \geqslant 1, n \in N$ and $0<\sum_{n=1}^{\infty} a_{n}^{p}<\infty, 0<\sum_{n=1}^{\infty} b_{n}^{r}<\infty$, then

$$
\sum_{n=1}^{\infty} \sum_{m=1}^{\infty} \frac{a_{m} b_{n}}{\max \left\{m^{\lambda}, n^{\lambda}\right\}}<\left\{\sum_{n=1}^{\infty}\left[\kappa(\lambda)-\frac{1}{3 r n^{\frac{r+\lambda-2}{r}}}\right] n^{1-\lambda} a_{n}^{p}\right\}^{\frac{1}{p}}\left\{\sum_{n=1}^{\infty}\left[\kappa(\lambda)-\frac{1}{3 p n^{\frac{p+\lambda-2}{p}}}\right] n^{1-\lambda} b_{n}^{r}\right\}^{\frac{1}{r}},
$$

where $k(\lambda)=\frac{p r \lambda}{(p+\lambda-2)(r+\lambda-2)}>0,2-\min \{p, r\}<\lambda \leqslant 2$. 
Proof. By (3.1) and $q>1$, we have

$$
\begin{aligned}
\sum_{n=1}^{\infty} \sum_{m=1}^{\infty} \frac{(q-1)^{\lambda} a_{m} b_{n}}{\max \left\{\left(q^{m}-1\right)^{\lambda},\left(q^{n}-1\right)^{\lambda}\right\}}< & \left\{\sum_{n=1}^{\infty}\left[\kappa(\lambda)+\mu(n)-\frac{1}{3 r n^{\frac{r+\lambda-2}{r}}}\right]\right. \\
& \left.\times n^{1-\lambda} a_{n}^{p}\right\}^{\frac{1}{p}}\left\{\sum _ { n = 1 } ^ { \infty } \left[\kappa(\lambda)+\mu(n)-\frac{1}{\left.\left.3 p n^{\frac{p+\lambda-2}{p}}\right] n^{1-\lambda} b_{n}^{r}\right\}^{\frac{1}{r}} .}\right.\right.
\end{aligned}
$$

So by (1.1), we have

$$
\begin{aligned}
\lim _{\mathrm{q} \rightarrow 1^{+}} & \sum_{n=1}^{\infty} \sum_{m=1}^{\infty} \frac{(q-1)^{\lambda} a_{m} b_{n}}{\max \left\{\left(q^{m}-1\right)^{\lambda},\left(q^{n}-1\right)^{\lambda}\right\}} \\
& =\lim _{q \rightarrow 1^{+}} \sum_{n=1}^{\infty} \sum_{m=1}^{\infty} \frac{a_{m} b_{n}}{\max \left\{\left(\frac{q^{m}-1}{q-1}\right)^{\lambda},\left(\frac{q^{n}-1}{q-1}\right)^{\lambda}\right\}} \\
& =\sum_{n=1}^{\infty} \sum_{m=1}^{\infty} \frac{a_{m} b_{n}}{\max \left\{m^{\lambda}, n^{\lambda}\right\}} \\
& <\lim _{q \rightarrow 1^{+}}\left\{\sum_{n=1}^{\infty}\left[\kappa(\lambda)+\mu(n)-\frac{1}{3 r n^{\frac{r+\lambda-2}{r}}}\right] n^{1-\lambda} a_{n}^{p}\right\}^{\frac{1}{p}}\left\{\sum_{n=1}^{\infty}\left[k(\lambda)+\mu(n)-\frac{1}{3 p n^{\frac{p+\lambda-2}{p}}}\right] n^{1-\lambda} b_{n}^{r}\right\}^{\frac{1}{r}} \\
& =\left\{\sum_{n=1}^{\infty}\left[\kappa(\lambda)-\frac{1}{3 r n^{\frac{r+\lambda-2}{r}}}\right] n^{1-\lambda} a_{n}^{p}\right\}^{\frac{1}{p}}\left\{\sum _ { n = 1 } ^ { \infty } \left[\kappa(\lambda)-\frac{1}{\left.\left.3 p n^{\frac{p+\lambda-2}{p}}\right] n^{1-\lambda} b_{n}^{r}\right\}^{\frac{1}{r}} \cdot}\right.\right.
\end{aligned}
$$

Hence the proof of the corollary is completed.

Remark 3.3. Inequality (3.1) is a q-analogue of the Hardy-Hilbert's inequality (1.5). Inequality (3.1) is a generalization of inequalities (1.3), (1.4), and (1.5).

\section{Acknowledgment}

This research is funded by Research Foundation of Chongqing University of Science and Technology, the project No. is ck2017zkyb019.

\section{References}

[1] G. Gasper, M. Rahman, Basic Hypergeometric Series, Cambrideg University Press, Cambrideg, (1990). 1

[2] G. H. Hardy, J. E. Littlewood, G. Polya, Inequalities, Cambrideg University Press, Cambridge, (1934). 1

[3] K. Jichang, L. Debnath, On new generalizations of Hilbert's inequality and their applications, J. Math. Anal. Appl., 245 (2000), 248-265. 1

[4] K. Knopp, Theory and application of infinite series, Blackie \& Son Limited, London, (1928). 2

[5] M. Krnic, J. Pecaric, General Hilbert's and Hardy's inequalities, Math. Inequal. Appl., 8 (2005), 29-51. 1

[6] J. Thomae, Beiträge zur Theorie der durch die Heinesche Reihe: darstellbaren Functionen, J. Reine Angew. Math., 70 (1869), 258-281. 1

[7] J. Thomae, Les séries Heinéennes supérieures, ou les séries de la forme, Annali di Matematica Pura ed Applicata, 4 (1870), 105-138. 1

[8] G. W. Xi, On generalizations and reinforcements of a Hilbert's type inequality, Rocky Mountain J. Math., 42 (2012), 329-337. 1

[9] G. W. Xi, A generalization of the Hilbert's type inequality, Math. Inequal. Appl., 18 (2015), 1501-1510. 1

[10] B. C. Yang, A new Hilbert-type inequality, Bull. Belg. Math. Soc. Simon Stevin, 13 (2006), 479-487. 1

[11] B. C. Yang, On a Strengthened version of the more accurate Hardy-Hilbert's inequality, Acta. Math. Sinica, 42 (1999), 1103-1110. 2

[12] B. C. Yang, On best extensions of Hardy-Hilbert's inequality with two parameters, JIPAM. J. Inequal. Pure Appl. Math., $6(2005), 15$ pages. 1

[13] B. C. Yang, Y. Zhu, Inequality on the Hurwitz Zeta-function restricted to the axis of positive reals, Acta Scientiarum Naturalium Universitatis Sunyatseni, 36 (1997), 30-35. 2 